\title{
BMC Hematology reviewer acknowledgement 2014
}

Nawsheen Boodhun

\section{Contributing reviewers}

The editors of BMC Hematology would like to thank all our reviewers who have contributed to the journal in Volume 14 (2014).

\section{Salem Abbes}

Tunisia

Omar Aljitawi

USA

Tanu Anand

India

Victoria Arija

Spain

Aranya Bagchi

USA

Samir K. Ballas

USA

Andrew Blann

UK

Kyle Bradley

USA

Carol Briggs

UK

Jo Caers

Belgium

Elena Cassinerio

Italy

Daniel Catovsky

UK

Harish Chandra

India

\author{
Ram K Chandyo \\ Nepal \\ Ying-Jun Chang \\ China \\ Annalisa Chiappella \\ Italy \\ Claudio Cimminiello \\ Italy \\ William Clark \\ Canada \\ Michael Clemente \\ USA \\ Philippe Connes \\ Guadeloupe \\ Alessandro Corso \\ Italy \\ Sharon Cox \\ UK \\ Anna Czyz \\ Poland \\ Wim De Kort \\ Netherlands \\ Masoud Dehdashtian \\ Iran \\ Enrico Derenzini \\ Italy
}

\author{
Ahmet Dogan \\ USA \\ Marco Paolo Donadini \\ Italy \\ Mehmet Ali Erkurt \\ Turkey \\ Lucia Farina \\ Italy \\ Jonathan Flanagan \\ USA \\ Ivan Gentile \\ Italy \\ Sophie Georgin-Lavialle \\ France \\ Thelma T Goncalez \\ USA \\ Pankaj Gupta \\ USA \\ Hans Erik Heier \\ Norway \\ Prashant Hiwarkar \\ UK \\ Alessandro Jenkner \\ Italy \\ Andrew Kerkhoff \\ USA
}

Correspondence: Nawsheen.Boodhun@biomedcentral.com

BioMed Central, Floor 6, 236 Gray's Inn Road, London WC1X 8HB, UK 


Michael Linden
USA
Chen Liu
USA
Elena Lukina
Russian Federation
Cuzzola Maria
Italy
Ismail Matalka
Jordan
Juan Mayordomo-Colunga
Spain
Saurabh Mehta
USA
Amha Mekasha
Ethiopia
Erin Meyer
USA
Gregorio Paolo Milani
Italy
Caterina Minniti
USA
Leonard Minuk
Canada
Daya Moodley
South Africa
Herman Nilsson-Ehle
Sweden
SA

Horatiu Olteanu

USA

Sjoukje Oosting

Netherlands

William Kwame Boakye

Ansah Owiredu

Ghana

Laura Palareti

Italy

Vincent Pialoux

France

Lisa Pieri

Italy

Kishor Raja

UK

Suely Rezende

Brazil

Vincent Ribrag

France

Paola Saracco

Italy

Marie P Schneider

Switzerland

Marie Scully

UK

David Snyder

USA

\author{
Anthony Staines \\ Ireland \\ Virginia Stallings \\ USA \\ Junko Tanaka \\ Japan \\ Kickler Tom \\ USA \\ Ankica Vasilj \\ Croatia \\ Lilly M Verhagen \\ Netherlands \\ Anders Vik \\ Norway \\ Jan Voorberg \\ Netherlands \\ Milena Vuica-Ross \\ USA \\ Dan Waxman \\ USA
}

Ambroise Wonkam

South Africa

Abdulraheem Yacoub
USA
C. Cameron Yin
USA
Binglan Yu
USA

